\title{
SHARP BOUNDS FOR EXPONENTIAL APPROXIMATIONS UNDER A HAZARD RATE UPPER BOUND
}

\author{
MARK BROWN, ${ }^{*}$ Columbia University
}

\begin{abstract}
Consider an absolutely continuous distribution on $[0, \infty)$ with finite mean $\mu$ and hazard rate function $h(t) \leq b$ for all $t$. For $b \mu$ close to 1 , we would expect $F$ to be approximately exponential. In this paper we obtain sharp bounds for the Kolmogorov distance between $F$ and an exponential distribution with mean $\mu$, as well as between $F$ and an exponential distribution with failure rate $b$. We apply these bounds to several examples. Applications are presented to geometric convolutions, birth and death processes, first-passage times, and to decreasing mean residual life distributions.
\end{abstract}

Keywords: Exponential approximation; hazard rate; Kolmogorov distance; geometric convolution; birth and death chain; first passage time; DMRL distribution

2010 Mathematics Subject Classification: Primary 60E15

Secondary 60K20; 60J27; 60J80; 90B25

\section{Introduction}

Consider an absolutely continuous distribution on $[0, \infty)$ with finite mean $\mu$ possessing a version of its hazard function $h(t)=f(t) / \bar{F}(t)$ satisfying $h(t) \leq b$ for all $t$. It appears plausible that if $b \mu$ is close to 1 , then $F$ should be approximately exponential. The purpose of this paper is to quantify this approximation. Examples will be discussed in Section 4, which show that the problem does arise naturally.

For approximating $F$ by an exponential distribution with mean $\mu$ (denoted by $\mu \&$ with $\&$ exponential with mean 1), we define $\delta=1-(b \mu)^{-1}$. The quantity $\delta$ is a scale-invariant measure of the proximity between $\mu$ and $b^{-1}$ (equivalently, between $b$ and $\mu^{-1}$ ). We would like to quantify the idea that small $\delta$ implies that $F$ is close to $\mu \varepsilon$. To measure proximity between distributions we employ the Kolmogorov distance

$$
D\left(F_{1}, F_{2}\right)=\sup \left|\bar{F}_{1}(t)-\bar{F}_{2}(t)\right|
$$

as well as its one-sided versions

$$
D^{+}\left(F_{1}, F_{2}\right)=\sup \left(\bar{F}_{1}(t)-\bar{F}_{2}(t)\right), \quad D^{-}\left(F_{1}, F_{2}\right)=D^{+}\left(F_{2}, F_{1}\right)=\sup \left(\bar{F}_{2}(t)-\bar{F}_{1}(t)\right) .
$$

In Proposition 2 (see Section 4) we derive our main results:

$$
\begin{gathered}
D^{+}(F, \mu \mathcal{E})=\sup \left(\bar{F}(t)-\mathrm{e}^{-t / \mu}\right) \leq 1-\mathrm{e}^{-\delta}, \\
D^{-}(F, \mu \mathcal{E})=\sup \left(\mathrm{e}^{-t / \mu}-\bar{F}(t)\right) \leq g(\delta)=\delta(1-\delta)^{(1-\delta) / \delta} .
\end{gathered}
$$

Both bounds (1) and (2) are sharp.

Received 5 December 2012; revision received 11 September 2014.

* Postal address: Department of Statistics, Columbia University, 1255 Amsterdam Avenue, New York, NY 10027, USA. Email address: mb2484@columbia.edu 
Thus, it follows that

$$
-\delta(1-\delta)^{(1-\delta) / \delta} \leq \bar{F}(t)-\mathrm{e}^{-t / \mu} \leq 1-\mathrm{e}^{-\delta} \text { for all } t \geq 0
$$

and

$$
D(F, \mu \mathcal{E})=\sup _{t}\left|\bar{F}(t)-\mathrm{e}^{-t / \mu}\right| \leq \max \left(1-\mathrm{e}^{-\delta}, g(\delta)\right) .
$$

The quantity on the right-hand side of (3) equals $1-\mathrm{e}^{-\delta}$ for $\delta \leq c \approx 0.8167$ and equals $g(\delta)$ for $\delta>c$. As we would only find the bounds useful for small $\delta$, the sharp upper bound for cases of practical interest is $1-\mathrm{e}^{-\delta}$.

As $b^{-1} \mathcal{E}$ and $\mu \mathcal{E}$ are close in Kolmogorov distance for small $\delta$, we could also approximate $F$ by $b^{-1} \mathcal{E}$. Since $F$ is stochastically larger than $b^{-1} \mathcal{E}, D\left(F, b^{-1} \mathscr{E}\right)$ and $D^{+}\left(F, b^{-1} \mathscr{E}\right)$ coincide. Our main result for this exponential approximation (see Proposition 1) is

$$
D\left(F, b^{-1} \mathcal{E}\right)=\sup \left(\bar{F}(t)-\mathrm{e}^{-b t}\right) \leq 1-\mathrm{e}^{-(b \mu-1)}=1-\mathrm{e}^{-(\delta /(1-\delta))} .
$$

The bound in (4) is sharp.

Exponential approximation under the constraint considered here does not appear to have been previously considered. In Section 4 we apply the methodology to obtain improved bounds in examples previously studied (geometric convolutions and first-passage times in time-reversible chains). In Example 3 of Section 5, we obtain a sharp bound for decreasing mean residual life (DMRL) distributions, based on knowledge of the first two moments as well as the hazard rate upper bound. It is hoped that these examples demonstrate that the bounds developed here are a useful addition to the methodology of exponential approximation.

\section{Preliminary results}

Since $\mu=b^{-1}$ implies that $F$ is exponential with mean $\mu$, a trivial case, we assume that $\mu>b^{-1}$.

Lemma 1. Fix $t=t_{1} \geq 0$ and $p$ in $[0,1]$. Consider the class of distributions with $\bar{F}\left(t_{1}\right)=$ $\mathrm{e}^{-b p t_{1}}$ and $\sup h(t) \leq b$. The stochastically smallest member of this class is given by

$$
\bar{F}_{0}(x)= \begin{cases}\mathrm{e}^{-b x}, & x \leq p t_{1}, \\ \mathrm{e}^{-b p_{1}}, & p t_{1}<x \leq t_{1}, \\ \mathrm{e}^{-b p t_{1}} \mathrm{e}^{-b\left(x-t_{1}\right)}, & x>t_{1} .\end{cases}
$$

Consequently, the smallest mean among distributions in this class is the mean of $F_{0}$,

$$
\mu_{0}\left(t_{1}, p\right)=\frac{1}{b}+q t_{1} \mathrm{e}^{-b t_{1} p} .
$$

For fixed $t_{1}, \mu_{0}\left(t_{1}, p\right)$ is strictly decreasing in $p$.

Proof. Let $F$ belong to the above class. We have

$$
\begin{gathered}
\bar{F}(x)=\mathrm{e}^{-\int_{0}^{x} h(s) \mathrm{d} s} \geq \mathrm{e}^{-\int_{0}^{x} b \mathrm{~d} s}=\mathrm{e}^{-b x}=\bar{F}_{0}(x) \quad \text { for } x \leq p t_{1}, \\
\bar{F}(x) \geq \bar{F}\left(t_{1}\right)=\mathrm{e}^{-b p t_{1}}=\bar{F}_{0}(x) \text { for } p t_{1}<x \leq t_{1},
\end{gathered}
$$

and

$$
\bar{F}(x)=\bar{F}\left(t_{1}\right)\left(\frac{\bar{F}(x)}{\bar{F}\left(t_{1}\right)}\right) \geq \mathrm{e}^{-b p t_{1}} \mathrm{e}^{-b\left(x-t_{1}\right)}=\bar{F}_{0}(x) \quad \text { for } x>t_{1} .
$$


Thus, $F$ is stochastically larger than $F_{0}$ for all $F$ in the class $\left(\sup h(t) \leq b, \bar{F}\left(t_{1}\right)=\mathrm{e}^{-b p t_{1}}\right)$, and $F_{0}$ belongs to this class as well. The rest of Lemma 1 now easily follows.

Lemma 2. Assume that $\sup h(t) \leq b$. Fix $t=t_{1}$ and $\mu>b^{-1}$. Then

$$
\bar{F}\left(t_{1}\right) \leq \mathrm{e}^{-b t_{1} p^{*}\left(t_{1}\right)} \quad \text { for } t_{1}>t_{0}=\mu-b^{-1},
$$

where $p^{*}\left(t_{1}\right)$ is the unique solution of

$$
q t_{1} \mathrm{e}^{-b p t_{1}}=\mu-\frac{1}{b}
$$

where $q=1-p$. The bound is sharp. For $t_{1} \leq t_{0}=\mu-b^{-1}$ the sharp upper bound for $\bar{F}\left(t_{1}\right)$ equals 1.

Proof. Consider the set of achievable pairs

$$
S=\left\{(p, \mu), \bar{F}\left(t_{1}\right)=\mathrm{e}^{-b p t_{1}}, \mu_{F}=\mu\right\}
$$

among distributions $F$ with $\sup h(t) \leq b$. By Lemma 1,

$$
\mu \geq \mu_{0}\left(t_{1}, p\right)=\frac{1}{b}+q t_{1} \mathrm{e}^{-b t_{1} p}
$$

and $\mu_{0}$ is strictly decreasing in $p$. Thus, for fixed $\mu>b^{-1}$ the section of $S$ at $\mu, S_{\mu}=$ $\{p:(p, \mu)$ belongs to $S\}$ consists of $p$ such that $\mu_{0}\left(t_{1}, p\right) \leq \mu$. This reduces to $\left[p^{*}\left(t_{1}\right), 1\right]$, where $p^{*}\left(t_{1}\right)$ is the unique solution to $\mu_{0}\left(t_{1}, p\right)=\mu$, equivalently, the unique solution to (6).

It follows that (5) holds for all $F$ with $\sup h(t) \leq b$ and $\mu_{F}=\mu_{0}\left(t_{1}, p\right)$. For each $t_{1}>\mu-b^{-1}$ the bound (5) is sharp. It is realized by the distribution

$$
\bar{F}^{*}(x)= \begin{cases}\mathrm{e}^{-b x}, & x \leq t_{1} p^{*}\left(t_{1}\right), \\ \mathrm{e}^{-b t_{1} p^{*}\left(t_{1}\right)}, & t_{1} p^{*}\left(t_{1}\right)<x \leq t_{1}, \\ \mathrm{e}^{-b t_{1} p^{*}\left(t_{1}\right)} \mathrm{e}^{-b\left(x-t_{1}\right)}, & x>t_{1} .\end{cases}
$$

Note that $F^{*}$ has mean $\mu$ with $\sup h(t) \leq b$ and achieves (5).

For $t \leq t_{0}=\mu-b^{-1}$, we cannot have a lower bound smaller than 1 . This is true because

$$
Z=\left(\mu-\frac{1}{b}\right)+\frac{\varepsilon}{b}
$$

with $\mathcal{E} \sim$ exponential(1) has mean $\mu$ with $\sup h(t) \leq b$, and $\bar{F}(t)=1$ for $t \leq \mu-b^{-1}$.

Lemma 3. For fixed $\mu>b^{-1}$ and $t>\mu-b^{-1}$, let $p^{*}(t)$ denote the solution to (6). Then both $t p^{*}(t)$ and $t q^{*}(t)$ are strictly increasing in $t \in\left(t_{0}, \infty\right)$.

Proof. Abbreviate $p^{*}(t)$ by $p^{*}$ and $q^{*}(t)$ by $q^{*}=1-p^{*}$. Denote the derivative of a function $f$ by $f^{\prime}$. By (6),

$$
\begin{gathered}
\left(q^{*} t\right)^{\prime}-b q^{*} t\left(p^{*} t\right)^{\prime}=0, \\
\left(q^{*} t\right)^{\prime}=\left(t-p^{*} t\right)^{\prime}=1-\left(p^{*} t\right)^{\prime} .
\end{gathered}
$$

Substituting (9) into (8), we obtain

$$
\left(p^{*} t\right)^{\prime}=\frac{1}{1+q^{*} t b}>0, \quad\left(q^{*} t\right)^{\prime}=\frac{q^{*} t b}{1+q^{*} t b}>0 .
$$


Lemma 4. Define $\hat{t}=b^{-1}+\mathrm{e}\left(\mu-b^{-1}\right)$ and $p^{*}$ as in Lemma 3. Then $p^{*}$ is strictly increasing in $\left(t_{0}, \hat{t}\right)$, achieves a maximum value of $(1+\mathrm{e}(b \mu-1))^{-1}$ at $\hat{t}$, and is strictly decreasing in $(\hat{t}, \infty)$. Each value of $p$ in $\left(0,(1+\mathrm{e}(b \mu-1))^{-1}\right)$ is achieved twice, once for a value $t \epsilon\left(t_{0}, \hat{t}\right)$ and once for a value $t>\hat{t}$.

Proof. Since $\left(t p^{*}\right)^{\prime}=p^{*}+t p^{*^{\prime}}$, it follows from (10) that

$$
p^{*^{\prime}}=\frac{q^{*}\left(1-b t p^{*}\right)}{t\left(1+b t q^{*}\right)} \text {. }
$$

As $t p^{*}$ is strictly increasing (see Lemma 3), it follows from (11) that $p^{*}$ is maximized at the unique value $\hat{t}$ for which $\hat{t} p^{*}(\hat{t})=b^{-1}$. Since $\hat{t} q^{*}(\hat{t})=\hat{t}-\hat{t} p^{*}(\hat{t})=\hat{t}-b^{-1}$, it follows from (6) that

$$
\mu-\frac{1}{b}=q^{*} \hat{t} \mathrm{e}^{-p^{*} b \hat{t}}=\frac{\hat{t}-b^{-1}}{\mathrm{e}}
$$

thus, $\hat{t}=b^{-1}+\mathrm{e}\left(\mu-b^{-1}\right)$ and

$$
p^{*}(\hat{t})=\frac{1}{(b \hat{t})}=\frac{1}{1+\mathrm{e}(b \mu-1)} .
$$

Since $p^{*}\left(t_{0}\right)=0$ the final claim will follow if we show that $\lim _{t \rightarrow \infty} p^{*}(t)=0$. Since $p^{*}$ is decreasing in $[\hat{t}, \infty)$ it has a limit $c \geq 0$. If $c$ were positive then $q^{*} t \mathrm{e}^{-b p^{*} t}$ would converge to 0 , in violation of (6). Thus, $c=0$. The next result follows from a routine differentiation argument. We record it here for use in Section 3.

Lemma 5. For $0<c<d<\infty$,

$$
\sup _{t \geq o}\left(\mathrm{e}^{-c t}-\mathrm{e}^{-d t}\right)=\left(1-\frac{c}{d}\right)\left(\frac{c}{d}\right)^{c /(d-c)}
$$

and is achieved at $\hat{t}=1 /(d-c) \log (d / c)$.

\section{Main results}

Proposition 1. Let $F$ have mean $\mu$ and $\sup h(t) \leq b$. Then

$$
D\left(F, b^{-1} \mathcal{E}\right)=\sup \left(\bar{F}(t)-\mathrm{e}^{-b t}\right) \leq 1-\mathrm{e}^{-(b \mu-1)} .
$$

The bound is sharp.

Proof. We have

$$
\bar{F}(t)-\mathrm{e}^{-b t} \leq 1-\mathrm{e}^{-b t} \leq 1-\mathrm{e}^{-b t_{0}}=1-\mathrm{e}^{-(b \mu-1)} \quad \text { for } t \leq t_{0}=\mu-b^{-1} .
$$

By (5),

$$
\bar{F}(t)-\mathrm{e}^{-b t} \leq \mathrm{e}^{-b p^{*} t}\left(1-\mathrm{e}^{-b q^{*} t}\right) \text { for } t>t_{0},
$$

where for each $t, p^{*}$ is the solution to (6) for that $t$, and $q^{*}=1-p^{*}$.

Since $t q^{*}(t)$ is increasing in $t$ (see Lemma 3$),\left(1-\mathrm{e}^{-x}\right) / x$ is decreasing in $x$, and $q^{*}\left(t_{0}\right)=1$, it follows from (6) and (12) that, for $t \geq t_{0}=\mu-b^{-1}$,

$$
\bar{F}(t)-\mathrm{e}^{-b t} \leq b q^{*} t \mathrm{e}^{-b p^{*} t}\left(\frac{1-\mathrm{e}^{-b q^{*} t}}{b q^{*} t}\right)=(b \mu-1)\left(\frac{1-\mathrm{e}^{-b t_{0}}}{b t_{0}}\right)=1-\mathrm{e}^{-(b \mu-1)} .
$$

To show sharpness, take $Z$ as in (7). Then $\mathbb{P}_{r}\left(Z>t_{0}\right)-\mathrm{e}^{-b t_{0}}=1-\mathrm{e}^{-(b \mu-1)}$. 
Proposition 2. Let $F$ be as in Proposition 1. Denote by $\mu \&$ an exponential distribution with mean $\mu$. Define $\delta=1-(b \mu)^{-1}$ and $g(\delta)=\delta(1-\delta)^{(1-\delta) / \delta}$. Then

(i) $D^{+}(F, \mu \mathcal{E})=\sup \left(\bar{F}(t)-\mathrm{e}^{-t / \mu}\right) \leq 1-\mathrm{e}^{-\delta}$;

(ii) $D^{-}(F, \mu \varepsilon)=\sup \left(\mathrm{e}^{-t / \mu}-\bar{F}(t)\right) \leq g(\delta)=\delta(1-\delta)^{(1-\delta) / \delta}$;

(iii) $D(F, \mu \mathcal{E})=\sup \left|\bar{F}(t)-\mathrm{e}^{-t / \mu}\right| \leq \max (\delta, g(\delta))= \begin{cases}1-\mathrm{e}^{-\delta}, & \delta \leq c, \\ g(\delta), & \delta>c,\end{cases}$ where $c \sim 0.8167$. The bounds in (i), (ii), and (iii) are sharp.

Proof. Recall that, from (5),

$$
\bar{F}(t)-\mathrm{e}^{-t / \mu} \leq \gamma(t) \triangleq \begin{cases}1-\mathrm{e}^{-t / \mu}, & t \leq t_{0}=\mu-b^{-1}, \\ \mathrm{e}^{-b p^{*} t}-\mathrm{e}^{-t / \mu}, & t>t_{0},\end{cases}
$$

where $p^{*}$ satisfies (6).

We first show that $\gamma(t)$ is decreasing in $\left(t_{0}, \hat{t}\right)$, where $\hat{t}=b^{-1}+e\left(\mu-b^{-1}\right)$. Now,

$$
\gamma^{\prime}(t)=\frac{\mathrm{e}^{-t / \mu}}{\mu}-b\left(p^{*} t\right)^{\prime} \mathrm{e}^{-b p^{*} t} .
$$

From (10) and (13), $\gamma^{\prime}(t)<0$ is equivalent to

$$
\mathrm{e}^{t\left(\mu^{-1}-b p^{*}\right)}>\frac{1+q^{*} t b}{b \mu}
$$

A sufficient condition for $\gamma^{\prime}(t)<0$ is, thus,

$$
1+t\left(\frac{1}{\mu}-b p^{*}\right)-\frac{1}{b \mu}-\frac{q^{*} t}{\mu}=\left(1-p^{*} b t\right) \delta>0 .
$$

For $t_{0}<t<\hat{t}, t p^{*}(t)<\hat{t} p^{*}(\hat{t})=b^{-1}$ (see Lemmas 3 and 4). Thus, (14) is positive and, consequently, $\gamma(t)$ is decreasing on $\left[t_{0}, \hat{t}\right)$. It follows that

$$
\bar{F}(t)-\mathrm{e}^{-t / \mu}=\gamma(t) \leq \gamma\left(t_{0}\right)=1-\mathrm{e}^{-\delta} \text { for } t_{0} \leq t \leq \hat{t} .
$$

If $t>\hat{t}$ then by Lemma 4 there exists $\tilde{t}$ in $\left(t_{0}, \hat{t}\right)$ with $p^{*}(\tilde{t})=p^{*}(t)$. Call this common value $p$ and let $q=1-p$. From (6), we have

$$
q \tilde{t} \mathrm{e}^{-b p \tilde{t}}=q t \mathrm{e}^{-b p t}
$$

Thus, from Lemma 5,

$$
p b=\frac{1}{t-\tilde{t}} \log \left(\frac{t}{\tilde{t}}\right)
$$

From (16) and Lemma 5,

$$
\mathrm{e}^{-b p \tilde{t}}-\mathrm{e}^{-b p t}=\sup _{\alpha \geq 0}\left(\mathrm{e}^{-\alpha \tilde{t}}-\mathrm{e}^{-\alpha t}\right)
$$

thus,

$$
\mathrm{e}^{b p \tilde{t}}-\mathrm{e}^{b p t} \geq \mathrm{e}^{-\tilde{t} / \mu}-\mathrm{e}^{-t / \mu}
$$

Equivalently,

$$
\gamma(t)=\mathrm{e}^{-b p t}-\mathrm{e}^{-t / \mu} \leq \mathrm{e}^{-p b \tilde{t}}-\mathrm{e}^{-\tilde{t} / \mu}=\gamma(\tilde{t}) .
$$


From (15) and (17),

$$
\bar{F}(t)-\mathrm{e}^{-t / \mu}=\gamma(t) \leq \gamma(\tilde{t}) \leq \gamma\left(t_{0}\right)=1-\mathrm{e}^{-\delta} \text { for } t>\hat{t}
$$

and Proposition 2(i) is proved. For sharpness, once again take $Z$ as defined in (7). Observe that

$$
\mathbb{P}\left(Z>t_{0}\right)-\mathrm{e}^{-t_{0} / \mu}=1-\mathrm{e}^{-\delta} .
$$

To derive Proposition 2(ii), note that

$$
\mathrm{e}^{-t / \mu}-\bar{F}(t) \leq \mathrm{e}^{-t / \mu}-\mathrm{e}^{-b t} \leq \delta(1-\delta)^{(1-\delta) / \delta}=g(\delta)
$$

by Lemma 5 . Sharpness follows by choosing $t_{2}=\mu /(b \mu-1) \log (b \mu), w=\mathrm{e}^{b t_{2}}\left(\mu-b^{-1}\right)$, and $F$ by

$$
\bar{F}(x)= \begin{cases}\mathrm{e}^{-b x}, & 0 \leq x \leq t_{2}, \\ \mathrm{e}^{-b t_{2}}, & t_{2}<x \leq t_{2}+w, \\ \mathrm{e}^{-b t_{2}} \mathrm{e}^{-b\left(x-\left(t_{2}+w\right)\right)}, & x>t_{2}+w .\end{cases}
$$

Then $F$ has mean $\mu, \sup h(t) \leq b$, and that $\bar{F}\left(t_{2}\right)=\mathrm{e}^{-b t_{2}}$. By Lemma 5 ,

$$
\mathrm{e}^{-t_{2} / \mu}-\mathrm{e}^{-b t_{2}}=g(\delta)
$$

and the bound is, thus, sharp. Finally, the statement that $g(\delta)<1-\mathrm{e}^{-\delta}$ for $\delta<c$ with the inequality reversing for $\delta>c$ comes from the numerical study of the function $f(x)=$ $1-\mathrm{e}^{-x}-g(x)$ for $0 \leq x \leq 1$.

\section{Examples}

Example 1. (Geometric convolutions.) Let $\left\{X_{i}\right\}$ be an independent and identically distributed (i.i.d.) sequence of nonnegative random variables with $v=\mathbb{E} X$ and $v_{2}=\mathbb{E} X^{2}<\infty$, where $\mathbb{E}$ denotes the expectation. Let $N$ be independent of $\left\{X_{i}\right\}$ with $\mathbb{P}_{r}(N=k)=q^{k} p, k=$ $0,1, \ldots, 0<p<1, q=1-p$, and define

$$
Y_{0}=\sum_{1}^{N} X_{i}
$$

The random variable $Y_{0}$ is known as a geometric convolution, or a compound geometric distribution, and arises naturally in many applied probability models. For example, a classic queueing theory result is that the stationary virtual waiting time distribution in a $G|G| 1$ queue can be represented as a geometric convolution. Köllerström [10] observed that the stationary waiting time distribution was new worse than used (NWU). Another relevant paper is Daley et al. [6]. As $Y_{0}$ is NWU,

$$
\mathbb{P}\left(Y_{0}>s+t\right) \geq \mathbb{P}\left(Y_{0}>s\right) \mathbb{P}\left(Y_{0}>t\right) \text { for all } s, t \geq 0 .
$$

Since $Y_{0}$ is NWU with finite mean $(q / p) v, Y_{0}$ is new worse than used in expectation (NWUE). It follows that $Y^{*}$, the equilibrium distribution corresponding to $Y_{0}$, has a hazard function $h^{*}$, which is bounded above by $\left(\mathbb{E} Y_{0}\right)^{-1}=p / q v$ for all $t$. Applying Propositions 1 and 2 with $b=p /(q v)$ and $\mu=\mathbb{E} Y^{*}=v_{2} / 2 v+q v / p$, it follows that

$$
D\left(Y^{*}, \frac{q v}{p} \mathcal{E}\right) \leq 1-\mathrm{e}^{-(b \mu-1)}=1-\mathrm{e}^{-\rho},
$$


where

$$
\rho=\left(\frac{\mathbb{E} Y_{0}^{2}}{2\left(\mathbb{E} Y_{0}\right)^{2}}\right)-1=\frac{p v_{2}}{2 q v^{2}}
$$

Furthermore,

$$
\begin{gathered}
D^{+}\left(Y^{*},\left(\mathbb{E} Y^{*}\right) \mathcal{E}\right) \leq 1-\mathrm{e}^{-(\rho / \rho+1)} \\
D^{-}\left(Y^{*},\left(\mathbb{E} Y^{*}\right) \mathcal{E}\right) \leq\left(\frac{\rho}{p+1}\right)\left(\frac{1}{\rho+1}\right)^{1 / \rho} \quad \text { for } \rho \leq c /(1-c) \approx 4.4569,
\end{gathered}
$$

and $D\left(Y^{*},\left(\mathbb{E} Y^{*}\right) \mathcal{E}\right) \leq 1-\mathrm{e}^{-(\rho / \rho+1)}$. Finally,

$$
D^{+}\left(Y_{0}, \frac{q v}{p} \varepsilon\right) \leq D\left(Y^{*}, \frac{q v}{p} \varepsilon\right) \leq 1-\mathrm{e}^{-\rho} .
$$

Results (18) and (19) improve upon $D\left(Y^{*},\left(\mathbb{E} Y^{*}\right) \mathscr{E}\right) \leq \rho$; see [12, p. 1396] and [11, p. 589]. Result (20) improves the bound in [4, p. 1396] from $\rho$ to $1-\mathrm{e}^{-\rho}$. Other approaches to geometric convolutions may be found in [4], [11], and [12].

Example 2. (Birth and death chain.) Consider $X_{1}, \ldots, X_{n}$ independent exponentially distributed random variables with failure rates $0<\lambda_{1} \leq \lambda_{2} \ldots, \leq \lambda_{n}<\infty$. Define $S=\sum_{1}^{n} X_{i}$. We have $S$ is the increasing failure rate (IFR) with limiting failure rate $\lambda_{1}$, thus, $h(t) \leq \lambda_{1}$ for all $t$. Now, $\mathbb{E} S=\sum_{1}^{n} \lambda_{i}^{-1}$. If $\lambda_{i}^{-1}$ is the dominant component of $\mathbb{E} S$ so that $\mathbb{E} S \approx \lambda_{1}^{-1}$, we would anticipate that $S$ is approximately exponential. The application of interest in this example is the first-passage time from 0 to $n$ in a continuous-time birth and death chain. Here, the $\lambda_{i}$ s are the eigenvalues of $-\tilde{Q}$, where $\tilde{Q}$ is the infinitesimal matrix restricted to $\{0, \ldots n-1\}$. For large $n$ these eigenvalues are often unavailable. However, $\mathbb{E} S$ can be computed from the birth and death rates (see [8, p. 148]) and $\lambda_{1}$ can be lower bounded by employing the extremal characterization of the smallest eigenvalue. In this case

$$
b=\lambda_{1}, \quad \mu=\sum_{1}^{n} \lambda_{i}^{-1}, \quad b \mu-1=\sum_{2}^{n} \frac{\lambda_{i}^{-1}}{\lambda_{1}^{-1}},
$$

while

$$
\delta=1-\left(\frac{1}{b \mu}\right)=\frac{\sum_{2}^{n} \lambda_{i}^{-1}}{\sum_{1}^{n} \lambda_{i}^{-1}} .
$$

It follows from Propositions 1 and 2 that $D\left(S, \lambda_{1}^{-1} \mathscr{E}\right) \leq 1-\mathrm{e}^{-(b \mu-1)}$,

$$
-\delta(1-\delta)^{(1-\delta) / \delta} \leq \mathbb{P}(S>t)-\mathrm{e}^{-t / \mathbb{E} S} \leq 1-\mathrm{e}^{-\delta} \quad \text { for all } t \geq 0 .
$$

These bounds offer an alternative to an approach of Soloviev [13] for birth and death chains. Soloviev's bound is based on

$$
\rho_{S}=1-\left(\frac{\mathbb{E} S^{2}}{2(\mathbb{E} S)^{2}}\right)
$$

and requires knowledge of $\sum_{1}^{n} \lambda_{i}^{-2}$.

The birth and death process first-passage time is nicely discussed in Gertsbakh [7]. 


\section{First-passage times}

For time-reversible finite state Markov chains, Keilson [9] showed that under general conditions the first-passage time to a set of states $A$, starting in steady state restricted to $A^{c}$, has a hazard function which is bounded above by its hazard at time 0 ,

$$
b=h(0)=\frac{\Pi\left(A^{c}\right)}{\sum_{j} \Pi_{j} q_{j A}},
$$

where $\Pi$ is the stationary distribution, $Q$ the infinitesimal matrix, and $q_{j A}=\sum_{k \epsilon A} q_{j k}$. Call this first-passage time $T$ and denote its mean by $\mu$. From Proposition 2, it follows that

$$
\sup _{t}\left|\mathbb{P}(T>t)-\mathrm{e}^{-t / \mu}\right| \leq 1-\mathrm{e}^{-\left[1-(h(0) \mu)^{-1}\right]} \quad \text { for } \delta=1-\left(\frac{1}{h(0) \mu}\right) \leq 0.8167 .
$$

This improves upon the bound of Brown [3, p. 422], lowering it from $\delta$ to $1-\mathrm{e}^{-\delta}$.

Importantly, we only use the property that $h_{T}(t) \leq h_{T}(0)$ for all $t$, while Keilson's approach employs complete monotonicity, the derivation of which requires spectral analysis for timereversible chains. This raises the hope of being able to quantify approximate exponentiality when time reversibility is not present. We plan to further explore this possibility in future work.

Example 3. Suppose that we know the first two moments of $F$ and have the hazard bound, $h \leq b$. How can this information be used to improve our bound for $D(F, \mu \mathcal{E})$ ? We do not have a general answer but we believe that the following example illustrates the potential of our methodology.

Assume that, in addition to knowledge of the first two moments (and $\sup h(t) \leq b$ ), $F$ is DMRL. The class which contains IFR as a subclass is defined by

$$
m(t)=\mathbb{E}(X-t \mid X>t)=\frac{\mu \bar{G}(t)}{\bar{F}(t)},
$$

nonincreasing in $t$, where $G$ is the equilibrium distribution corresponding to $F$. For new better than used in expectation (NBUE) distributions (of which DMRL is a subclass), Brown [5, p. 206] derived the following result:

$$
\bar{F}(t) \leq \mathrm{e}^{1-\mu^{-1} m(t)} \mathrm{e}^{-t / \mu}
$$

Thus,

$$
\bar{F}(t)-\mathrm{e}^{-t / \mu} \leq\left[1-\mathrm{e}^{-\left[1-\mu^{-1} m(t)\right]}\right] \bar{F}(t) .
$$

Next, define $L(t)=1-\left(\mu^{-1} m(t)\right)^{2}$ and note that for $F$ DMRL, $L$ is a nondecreasing function. Moreover,

$$
\mathbb{E} L(X)=1-\left(\frac{\mathbb{E} m^{2}(X)}{\mu^{2}}\right)=1-\left(\frac{\sigma^{2}}{\mu^{2}}\right),
$$

where we have used the well known identity, $\mathbb{E} m^{2}(X)=\sigma^{2}$ of Bremaud [2].

Unless $F$ is exponential there exists a number $t_{1} \geq 0$ such that $m(t)<\mu$ for all $t>t_{1}$, and $\bar{F}(t)=\mathrm{e}^{-t / \mu}$ for all $t \leq t_{1}$. For $t>t_{1}$, applying Markov's inequality and (22), we obtain

$$
\bar{F}(t) \leq \frac{\mathbb{E} L(X)}{L(t)}=\frac{1-\left(\sigma^{2} / \mu^{2}\right)}{1-(m(t) / \mu)^{2}} .
$$


From (21) and (23), we have

$$
\bar{F}(t)-\mathrm{e}^{-t / \mu} \leq \frac{\left(1-\left(\sigma^{2} / \mu^{2}\right)\right)\left(1-\mathrm{e}^{-\left(1-\mu^{-1} m(t)\right)}\right)}{1-(m(t) / \mu)^{2}} .
$$

Let $x=1-(m(t) / \mu)$ and note that $x$ ranges from 0 to $1-(\mu b)^{-1}=\delta$, as follows from $m(0)=\mu$ and $\sup h(t) \leq b$. The function

$$
r(x)=\frac{1-\mathrm{e}^{-x}}{x(2-x)}
$$

increases from $\frac{1}{2}$ to $\left(1-\mathrm{e}^{-\delta}\right) / \delta(2-\delta)$ as $x$ increases from 0 to its maximum value of $\delta$.

Thus, from (24), we have

$$
\sup _{t}\left(\bar{F}(t)-\mathrm{e}^{-t / \mu}\right) \leq\left(1-\left(\frac{\sigma^{2}}{\mu^{2}}\right)\right)\left(\frac{1-\mathrm{e}^{-\delta}}{\delta(2-\delta)}\right) .
$$

Note that $\mathrm{e}^{-t / \mu}-\bar{F}(t) \leq \mathrm{e}^{-t / \mu}-\bar{G}(t)$ and (see [4, p. 1390]) for $F$ NBUE, we have

$$
\sup _{t}\left(\mathrm{e}^{-t / \mu}-\bar{G}(t)\right) \leq \frac{1}{2}\left(1-\frac{\sigma^{2}}{\mu^{2}}\right) \leq \frac{1-\mathrm{e}^{-\delta}}{\delta(2-\delta)}\left(1-\frac{\sigma^{2}}{\mu^{2}}\right) .
$$

Thus, from (25) and (26),

$$
D(F, \mu \varepsilon) \leq\left(1-\left(\frac{\sigma^{2}}{\mu^{2}}\right)\right) \frac{1-\mathrm{e}^{-\delta}}{\delta(2-\delta)} .
$$

We now argue that (27) is sharp. Note that, since $\sigma^{2}=\mathbb{E} m^{2}(X) \geq 1 / b^{2}$,

$$
1-\left(\frac{\sigma^{2}}{\mu^{2}}\right) \leq 1-\left(\frac{1}{b \mu^{2}}\right)=\delta(2-\delta)
$$

where $\delta=1-(b \mu)^{-1}$. Also note that if $F$ is NBUE and not exponential then $\sigma<\mu$.

Given $\left(\mu, b, \sigma^{2}\right)$ with $\mu>b^{-1}, \sigma<\mu$, and (30) holding, we will construct a DMRL distribution $F$ having the desired $\left(\mu, b, \sigma^{2}\right)$ and achieving the bound in (27). For a fixed value $s \geq 0$, define

$$
\bar{F}_{s}(x)= \begin{cases}\mathrm{e}^{-x / \mu}, & 0 \leq x \leq s, \\ \mathrm{e}^{-s / \mu}, & s<x \leq s+\mu-b^{-1}, \\ \left.\mathrm{e}^{-s / \mu} \mathrm{e}^{-b\left(x-\left(s+\mu-b^{-1}\right)\right.}\right), & x>s+\mu-b^{-1} .\end{cases}
$$

First we check that the following two properties hold and then use them in (iii) (below) to show that the bound in (27) is attained.

(i) $F_{s}$ is DMRL and has mean $\mu$. This follows since $m(x)=\mu$ for $0 \leq x \leq s, m(x)=$ $s+\mu-x<\mu$ for $s<x \leq s+\mu-b^{-1}$, and $m(x)=b^{-1}<\mu$ for $x>s+\mu-b^{-1}$.

(ii) $\mu_{2}=\mathbb{E} X^{2}=\int_{0}^{\infty} 2 t \bar{F}(t) \mathrm{d} t=2 \mu^{2}-\left[\mu^{2}-b^{-2}\right] \mathrm{e}^{-s / \mu}$ and, thus, $1-\left(\sigma_{s}^{2} / \mu^{2}\right)=$ $\left(1-(b \mu)^{-2}\right) \mathrm{e}^{-s / \mu}=\delta(2-\delta) \mathrm{e}^{-s / \mu}$, where $\sigma_{s}^{2}$ is the variance corresponding to $F_{s}$. Given $\left(\mu, b, \sigma^{2}\right)$ with $1-\left(\sigma^{2} / \mu^{2}\right) \leq \delta(2-\delta)$, we choose $s \geq 0$ so that

$$
\mathrm{e}^{-s / \mu}=\frac{1-\left(\sigma^{2} / \mu^{2}\right)}{\delta(2-\delta)}
$$

For this choice of $s$, the distribution $F_{s}$ is DMRL with the specified values of $\left(\mu, b, \sigma^{2}\right)$ (since $\sigma_{s}^{2}=\sigma^{2}$ ). 
(iii) To show that $D\left(F_{s}, \mu \mathcal{E}\right)$ attains the upper bound in (27) for this choice of $s$, note that

$$
\bar{F}_{s}\left(s+\mu-\frac{1}{b}\right)-\mathrm{e}^{-\left(s+\mu-b^{-1}\right) / \mu}=\mathrm{e}^{-s / \mu}\left(1-\mathrm{e}^{-\delta}\right)=\left(1-\frac{\sigma^{2}}{\mu^{2}}\right)\left(\frac{1-\mathrm{e}^{-\delta}}{\delta(2-\delta)}\right)
$$

follows from (29).

Thus, the upper bound in (27) is achieved.

Example 4. Suppose that $X \sim F=\Gamma(1.1,1)$, so that

$$
f(x)=(\Gamma(1.1))^{-0.1} x^{-0.1} \mathrm{e}^{-x}, \quad x>0,
$$

$F$ is IFR and, thus, DRML. In this example $\mu=\sigma^{2}=1.1$ and $b=1$. Consequently,

$$
\delta=\frac{1}{11}, \quad 1-\frac{\sigma^{2}}{\mu^{2}}=\frac{1}{11} .
$$

From Proposition 2, the bound for $D(F, \mu \mathcal{E})$, which uses $\mu$ and $b$ but not $\sigma^{2}$ provides the following bound:

$$
D(F, \mu \varepsilon) \leq 1-\mathrm{e}^{-\delta} \approx 0.08690
$$

A bound which utilizes $\mu$ and $\sigma^{2}$ but ignores $b$ would be (27) with $b=\infty$ and $\delta=1$, namely,

$$
D(F, \mu \varepsilon) \leq\left(1-\frac{\sigma^{2}}{\mu^{2}}\right)\left(1-\frac{1}{\mathrm{e}}\right) \approx 0.05747 .
$$

Finally, the bound in (27) (which makes use of all three parameters (i)-(iii)) yields

$$
D(F, \mathcal{E}) \leq\left(1-\frac{\sigma^{2}}{\mu^{2}}\right)\left(\frac{1-\mathrm{e}^{-\delta}}{\delta(2-\delta)}\right) \approx 0.04552
$$

\section{References}

[1] Barlow, R. E. and Proschan, F. (1975). Statistical Theory of Reliability and Life Testing: Probability Models. Holt, Rinehart and Winston, New York.

[2] Brémaud, P. (1981). Point Processes and Queues: Martingale Dynamics. Springer, New York.

[3] Brown, M. (1983). Approximating IMRL distributions by exponential distributions, with applications to first passage times. Ann. Prob. 11, 419-427.

[4] Brown, M. (1990). Error bounds for exponential approximations of geometric convolutions. Ann. Prob. 18, 1388-1402.

[5] Brown, M. (2006). Exploiting the waiting time paradox: applications of the size-biasing transformation. Prob. Eng. Inf. Sci. 20, 195-230.

[6] Daley, D. J., KreǏnin, A. YA, And Trengove, C. D. (1992). Inequalities concerning the waiting-time in single-server queques: a survey. In Queueing and Related Models, Oxford University Press, pp. 177-223.

[7] Gertsbakh, I. B. (1989). Statistical Reliability Theory. Marcel Dekker, New York.

[8] Karlin, S. and Taylor, H. M. (1975). A First Course in Stochastic Processes, 2nd edn. Academic Press, New York.

[9] KeILson, J. (1979). Markov Chain Models—Rarity and Exponentiality. Springer, New York.

[10] Köllerström, J. (1976). Stochastic bounds for the single-server queue. Math. Proc. Cambr. Phil. Soc. 80, 521-525.

[11] Peköz, E. A. ANd Röllin, A. (2011). New rates for exponential approximation and the theorems of Rényi, and Yaglom. Ann. Prob. 39, 587-608.

[12] Soloviev, A. (1971). Asymptotic behaviour of the time of first occurrence of a rare event. Eng. Cybernetics 9 , $1038-1048$.

[13] Soloviev, A. D. (1972). Asymptotic distribution of the moment of first crossing of a high level by a birth and death process. In Proceedings of the Sixth Berkeley Symposium on Mathematical Statististics and Probability, Volume III, Probability Theory, University of California Press, Berkely, pp. 71-86. 